\title{
Phenytoin crystal growth rates in the presence of phosphate and chloride ions
}

\author{
G.L. Zipp and N. Rodríguez-Hornedo ${ }^{1}$ \\ College of Pharmacy, The University of Michigan, Ann Arbor, Michigan 48109-1065, USA
}

Received 6 January 1992; manuscript received in final form 16 March 1992

\begin{abstract}
Phenytoin crystal growth kinetics have been measured as a function of supersaturation in $\mathrm{pH} 2.2$ phosphoric acid and $\mathrm{pH} 2.2$ hydrochloric acid solutions. Two different methods were used for the kinetic analysis. The first involved a zone-sensing device which provided an analysis of the distribution of crystals in a batch crystallizer. Crystal growth rates were calculated from the increase in the size of the distribution with time. In the second method, growth rates were evaluated from the change in size with time of individual crystals observed under an inverted microscope. The results from each method compare favorably. The use of both techniques provides an excellent opportunity to exploit the strengths of each: an average growth rate from a population of crystals from batch crystallization and insight into the effect of growth on the morphology of the crystals from the individual crystal measurements.
\end{abstract}

\section{Introduction}

Phenytoin, 5-5-diphenylhydantoin (DPH), is a widely used anticonvulsant, effective in the control and treatment of epilepsy. Its intravenous administration is greatly complicated by the risk of precipitation of the free acid [1-3]; either after dilution of the injection solution into intravenous fluids, or at the site of injection. DPH is similar to many other pharmaceutical compounds in that its low aqueous solubility can result in precipitation of the drug which affects uptake, blood levels and bioavailability. Thus, it is expedient to understand the crystallization of these compounds with the ultimate goal of formulating non-precipitating or predictably precipitating dosage forms. Toward this end, the growth kinetics of DPH crystals have been studied in two solvents; $\mathrm{pH} 2.2$ phosphoric acid and $\mathrm{pH} 2.2$ hydrochloric acid solutions.

\footnotetext{
I To whom correspondence should be addressed.
}

\section{Experimental procedure}

\subsection{Materials}

All chemicals used were reagent grade. Both acid solutions were made from filtered distilled de-ionized water by adding $0.10 \mathrm{M} \mathrm{NaOH}$ to $0.10 \mathrm{M}$ acid solution $\left(\mathrm{HCl}\right.$ or $\left.\mathrm{H}_{3} \mathrm{PO}_{4}\right)$ until the $\mathrm{pH}$ was 2.2. The ionic strength was then adjusted to 0.15 with the addition of $\mathrm{NaCl}$.

\subsection{Creation of supersaturation and seed crystals}

The solubility of DPH [4] is a strong function of $\mathrm{pH}$ (fig. 1). In both experimental procedures, a $\mathrm{pH}$ change was used to nucleate seed crystals and to produce a supersaturated solution. This was done by adding a small portion of a high DPH concentration $(2-10 \mathrm{mg} / \mathrm{g}) 0.1 \mathrm{M} \mathrm{NaOH}$ solution to the solvent of choice. Nucleation of phenytoin crystals occurred immediately upon contact between the $\mathrm{DPH} / \mathrm{NaOH}$ solution and the solvent. However, the number of crystals formed was not sufficient to completely deplete the excess DPH- 


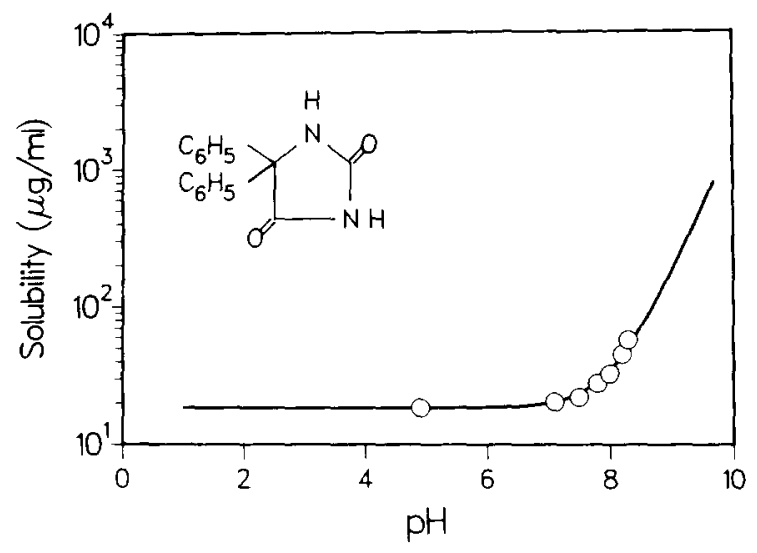

Fig. 1. Phenytoin solubility as a function of $\mathrm{pH}$ in phosphate buffer at $25^{\circ} \mathrm{C}$ (after ref. [4]).

from solution, and the seed crystals then grew from the residual supersaturation. The number of crystals produced could be controlled by varying the DPH concentration of the DPH/NaOH solution; a high DPH concentration $(8-12 \mathrm{mg} / \mathrm{g})$ would produce a large number of seeds, while a lower concentration $(2-4 \mathrm{mg} / \mathrm{g}$ ), would cause very few crystals to nucleate.

\subsection{Batch crystallization experiments}

These were carried out in situ with a Coulter Counter Multisizer. A contoured bottom flask was used as the crystallization vessel. It was filled with a known amount of solvent and the agitation rate of the impeller was set at $400 \mathrm{rpm}$. Nucleation of seed crystals and creation of supersaturation were initiated by adding a small amount of $\mathrm{DPH} / \mathrm{NaOH}$ solution to the vessel. The crystal size distribution (CSD) in the vessel was then measured throughout the experiment using a 140 $\mu \mathrm{m}$ orifice. The phenytoin concentration profile was also monitored by measuring the UV absorbance of a filtered, diluted aliquot of the crystallizer slurry.

\subsection{Flow cell experiments}

Single crystal growth rates were determined by measuring the increase in crystal size with time under an inverted microscope, at constant super- saturation. Details of the flow system used are presented elsewhere [5]. Seed crystals were produced by diluting a $\mathrm{DPH} / \mathrm{NaOH}$ solution into the solvent as in the batch experiments. After nucleation, approximately $1 \mathrm{ml}$ of the slurry was placed into a flow cell and the flow cell mounted onto the microscope stage. The seed crystals introduced into the cell were allowed to settle to the bottom of the cell and remained in the same location during growth.

A growth solution was then made by adding a more dilute $\mathrm{DPH} / \mathrm{NaOH}$ solution to the buffer. A small number of phenytoin crystals would nucleate upon production of the growth solution but their growth did not significantly reduce the concentration of the solution. The growth solution was pumped through the cells at a constant velocity $(0.07$ to $0.28 \mathrm{~cm} / \mathrm{s})$ and growth rate measurements started after a volume equal to the cell volume had been pumped.

The image from the microscope was displayed on a separate monitor. The outline of several crystals from each cell was drawn at several time intervals and the crystal dimensions (length and width) calculated from the outline and the appropriate conversion factors (obtained with the use of a calibration slide). The concentration of the growth solution was monitored with time to assure that it remained constant.

\section{Results and discussion}

\subsection{Batch crystallization experiments}

A sample of measured CSDs from a batch crystallization experiment is presented in fig. 2 . The first measurement of the CSD at 2 min (the time elapsed after addition of the DPH solution) shows that only a small portion of the CSD has grown larger than the minimum size measurable by the orifice. As time progresses, continued growth of the crystals results in more of the CSD growing larger than $8 \mu \mathrm{m}$ until, at $3.5 \mathrm{~min}$, the entire CSD is visible. After this time, the seed crystals continue to grow (as evidenced by the rightward shift of the CSD) while no additional crystals are seen. 
Fig. 3 presents the sample of CSDs shown in fig. 2 as cumulative number profiles. Phenytoin growth rates were calculated from the change in the cumulative number distribution with time [6]. Fig. 4 displays the DPH concentration profile measured during the experiment. As was typical of most DPH batch experiments, the concentration changed only slightly between CSD measurements and thus a linear interpolation between data points was used to determine the supersaturation corresponding to a given growth rate measurement.

\subsection{Flow cell experiments}

Phenytoin crystals grow as slightly elongated rods (fig. 5). The orientation of the crystal axes with respect to the observed morphology was found from single crystal X-ray analysis; the long dimension is parallel to the $\boldsymbol{a}$ axis and the width is parallel to the $c$ axis. The average growth rate

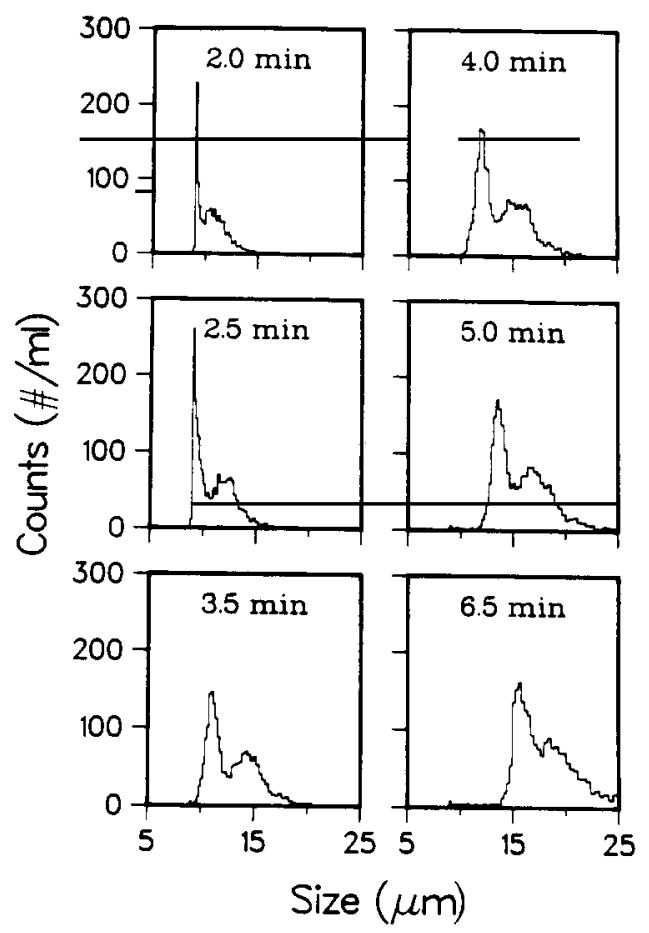

Fig. 2. Differential plots of measured crystal size distribution.

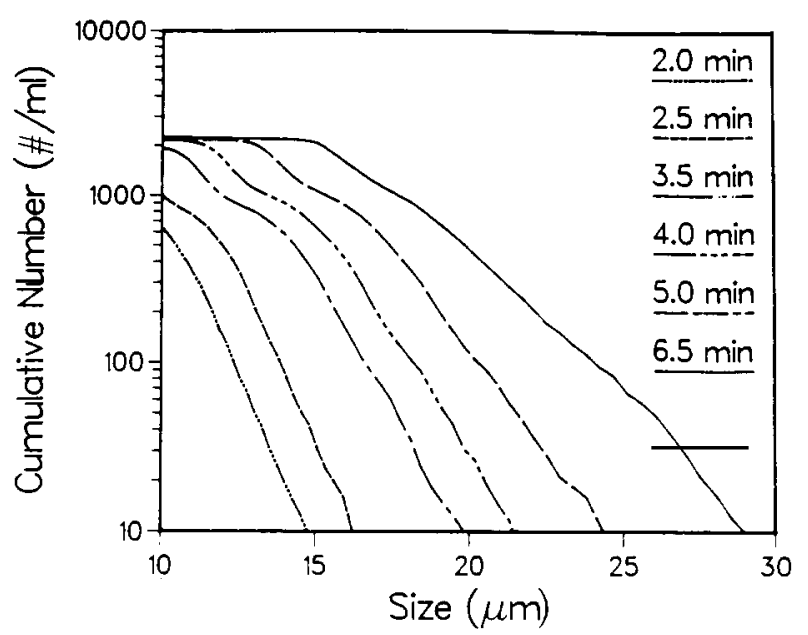

Fig. 3. Cumulative number plot of measured crystal size distribution.

of the long dimension, $\bar{G}_{a}$, and the width, $\bar{G}_{c}$, the standard deviation of the average and the number of crystals measured can be found in table 1 . These values are presented as a function of supersaturation in fig. $6 \mathrm{a}$ (phosphoric acid solution) and fig. 6b (hydrochloric acid solution). In both solvents, the growth rate in the $a$ direction is faster and has a stronger dependence upon supersaturation than the growth rate in the $c$ direction. A comparison of the measured growth rates in the two solvents (fig. 7) reveals that the growth rate in the $a$ direction is only slightly affected by solvent composition (fig. 7a). However, at supersaturation levels higher than 0.9 , crystal growth in the $c$ direction is faster in the phosphoric acid solution than in the hydrochloric acid solution (fig. 7b).

Phenytoin crystal morphology can be affected by the choice of solvent and the supersaturation under which the crystals are grown. The ratio of growth rates in the two directions measured, $\bar{G}_{a} / \bar{G}_{c}$, versus supersaturation is shown in fig. 8 . The ratio does not depend upon supersaturation for crystals grown in phosphoric acid solution. Thus, the overall shape of a crystal would be maintained during growth in this supersaturation range. However, for crystals grown in hydrochloric acid solution, the ratio of $\bar{G}_{a}$ to $\bar{G}_{c}$ does 


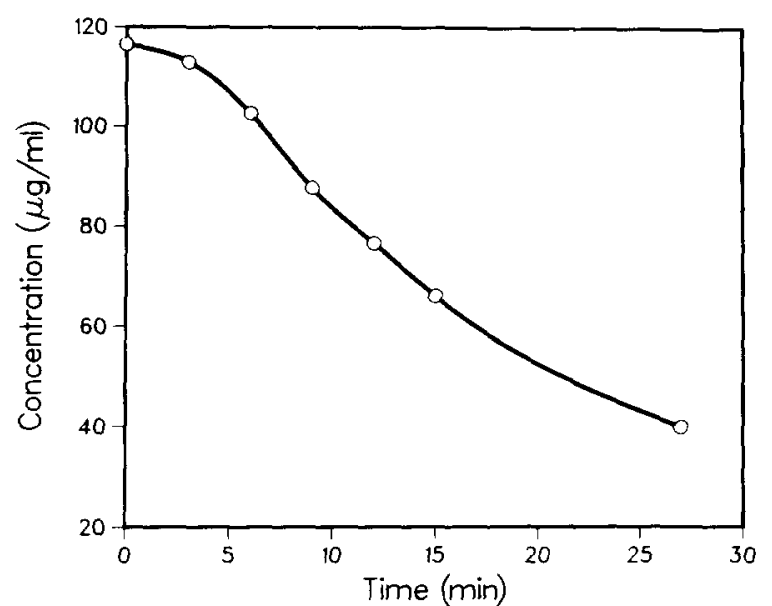

Fig. 4. Phenytoin concentration profile measured in a batch crystallization experiment.

increase with supersaturation and the crystals would then be expected to become more elongated at higher supersaturation.

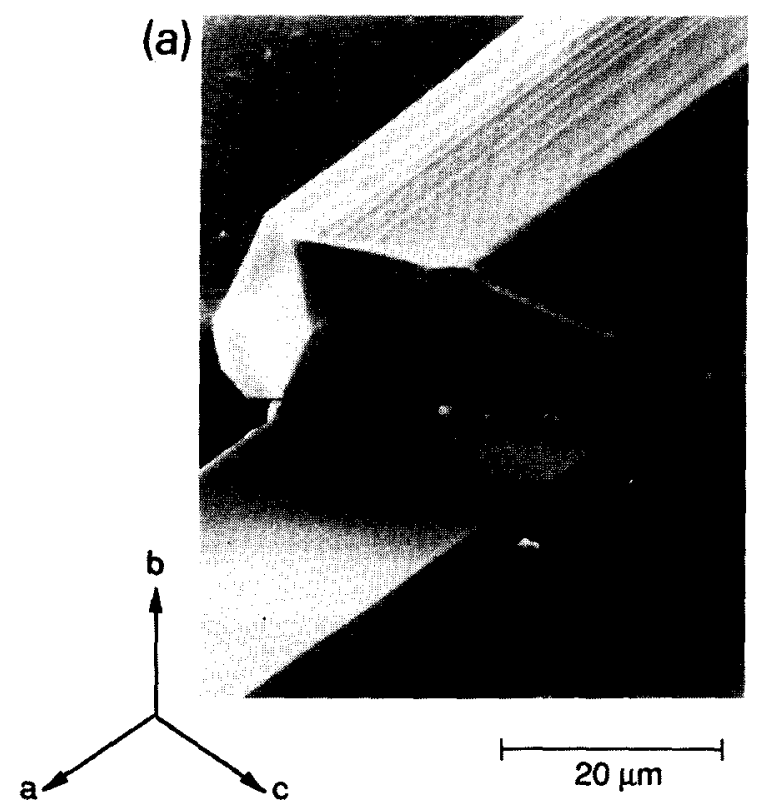

\subsection{Comparison of methods}

Phenytoin crystal growth rates measured in batch experiments (multiparticulate system), and growth rates in the $a$ direction measured in flow cell experiments (single crystals), are shown as a function of supersaturation in fig. 9. From these results it can be concluded that the growth rates measured with the two methods agree well, despite the different representation of crystal size obtained from each method. Because the batch experiments were conducted with a zone-sensing device, the length measurement used to calculate growth rates was the diameter of a sphere with a volume equivalent to the volume of the particle rather than the actual crystal length as found in the individual crystal measurements. However, the single crystal growth rate measurements were all obtained at fairly low supersaturation, where growth in all three directions may be expected to be of the same order of magnitude. Thus, under these conditions, growth in one direction only

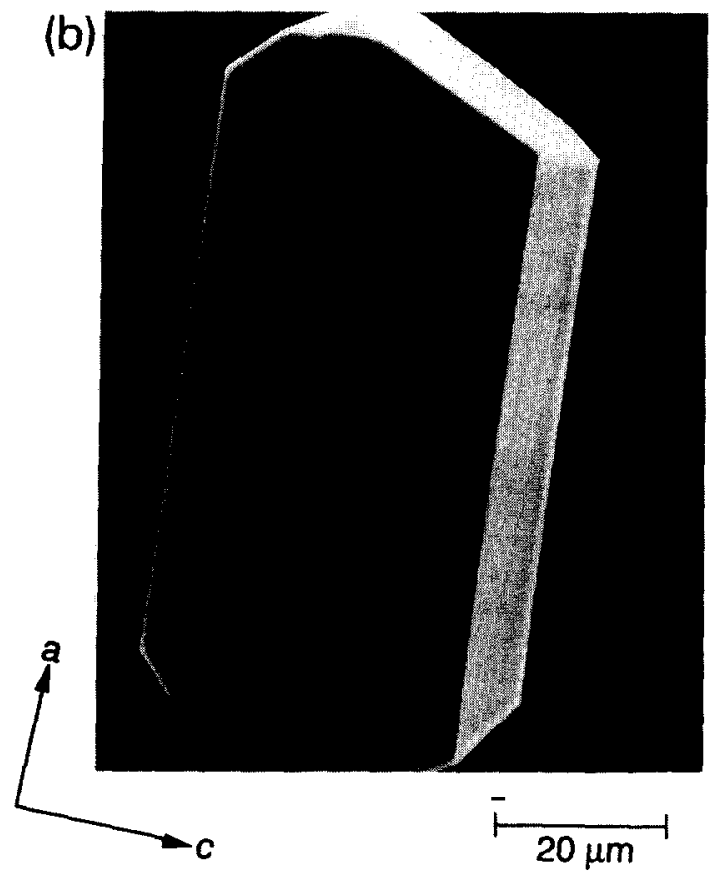

Fig. 5. Scanning electron micrographs of phenytoin crystals grown in flow cell experiments: (a) phosphoric acid solution; (b) hydrochloric acid solution looking directly down the $b$-axis. 
may be sufficient to characterize overall growth rates.

\subsection{Solvent effects}

The dependence of phenytoin crystal growth rate on supersaturation can be described by a power law model as:

$G=k_{\mathrm{g}} \sigma^{a}$,

where $G$ is the growth rate, $k_{\mathrm{g}}$ is the growth rate constant, $\sigma$ is the supersaturation and $a$ is the supersaturation exponent. The supersaturation,
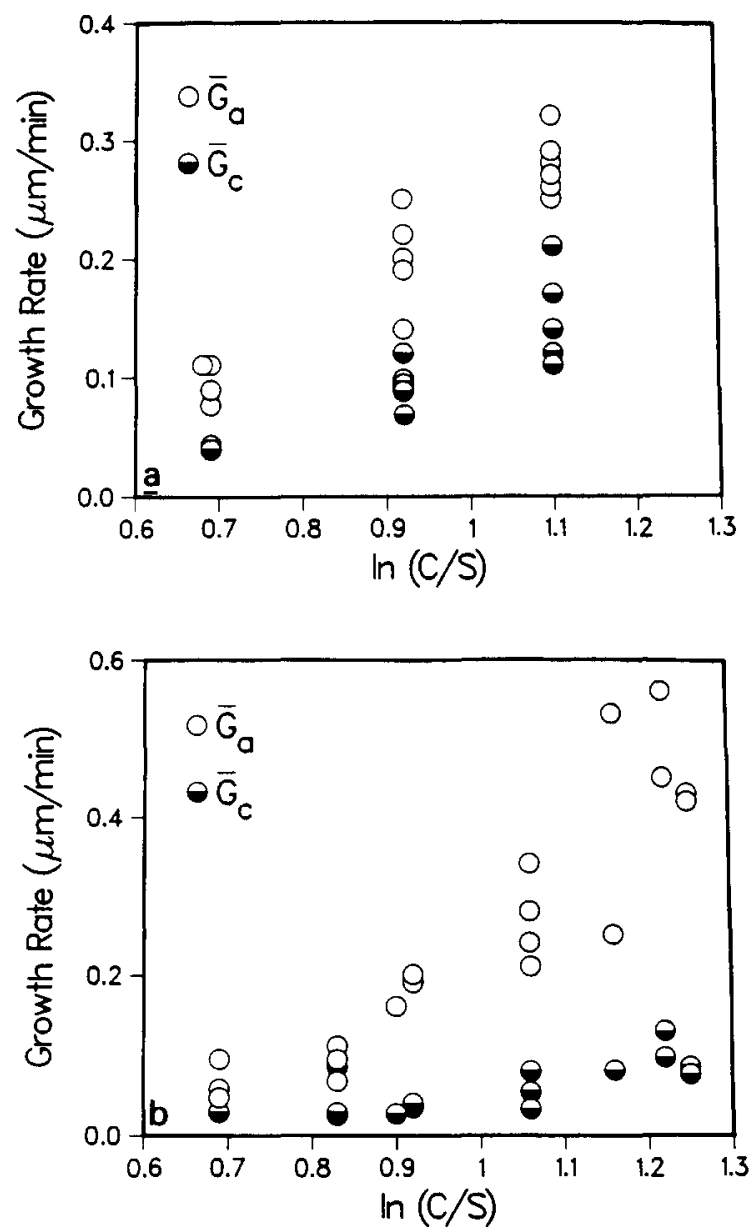

Fig. 6. Phenytoin crystal growth rates in the $a$ and $c$ directions plotted as a function of supersaturation: (a) phosphoric acid solution; (b) hydrochloric acid solution.
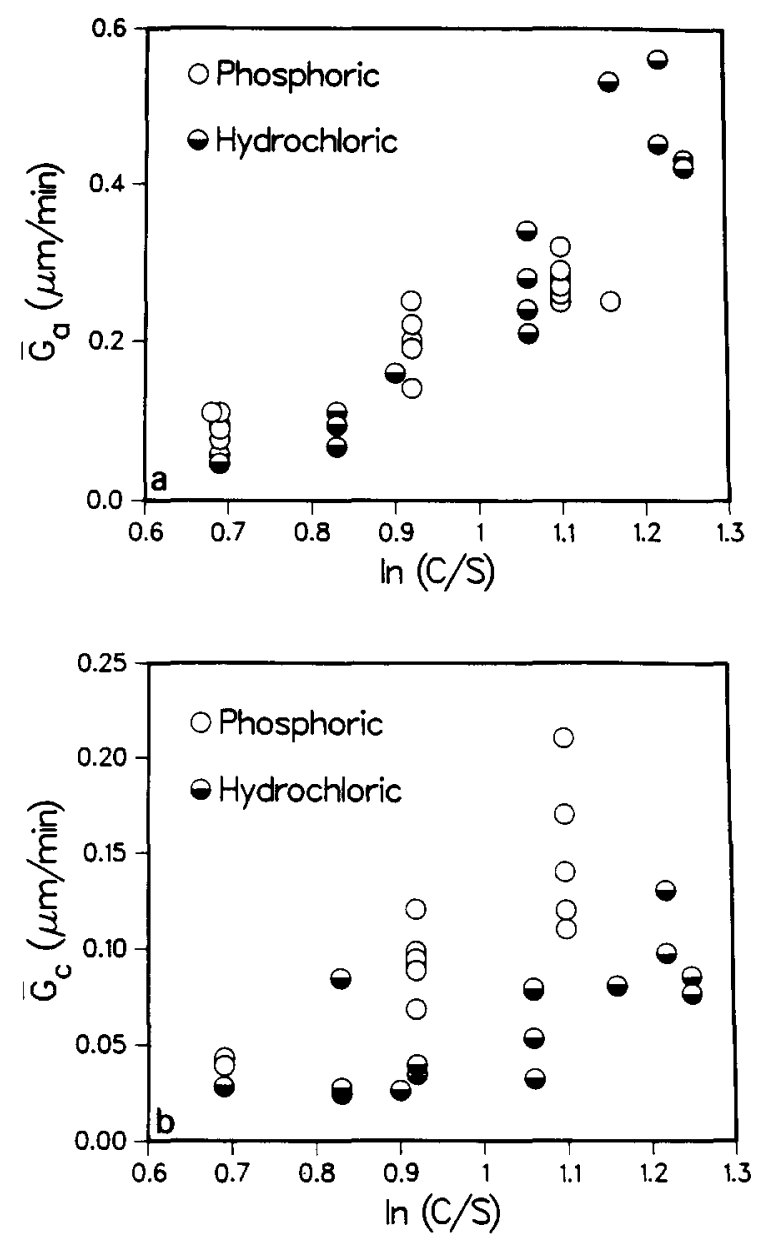

Fig. 7. Comparison of phenytoin crystal growth rates measured in the two solvents: (a) growth in the a direction; (b) growth in the $c$ direction.

$\sigma$, is defined as $\ln (C / S)$, where $C$ is the phenytoin concentration and $S$ is the phenytoin solubility at the crystallization conditions. The parameters $k_{\mathrm{g}}(\mu \mathrm{m} / \mathrm{min})$ and $a$ were evaluated from a linear regression of $\ln G$ versus $\ln \sigma$. Their values are presented in table 2 and were used to draw the solid lines shown in fig. 9.

The decrease of the supersaturation exponent, $a$, from 3.7 in hydrochloric acid solution to 3.3 in phosphoric acid solution and the different dependence of growth rate ratios, $\bar{G}_{a} / \bar{G}_{c}$, on supersaturation, found in the two solvents may indicate a change in the growth mechanism with solvent. A 
change in mechanism is supported by the contrast of the surface appearance of crystals grown from the two solutions. The crystal faces that contribute to growth in the $a$ direction are smooth on crystals grown from both solvents (see fig. 5).
However, the faces that contribute to growth in the $c$ direction are much rougher on crystals grown in phosphoric acid (fig. Sa) than are those on crystals grown in hydrochloric acid (fig. 5b). The morphology also supports the measured

Table 1

Average growth rates in the $a$ and $c$ directions, their standard deviations and the number of crystals observed in the flow cell experiments

\begin{tabular}{|c|c|c|c|c|c|c|c|c|}
\hline Run & Cell & $\begin{array}{l}\sigma= \\
\ln (C / S)\end{array}$ & $\begin{array}{l}\bar{G}_{a} \\
(\mu \mathrm{m} / \mathrm{min})\end{array}$ & SD & $n$ & $\begin{array}{l}\bar{G}_{c} \\
(\mu \mathrm{m} / \mathrm{min})\end{array}$ & SD & $n$ \\
\hline \multicolumn{9}{|c|}{ Phosphoric acid solution } \\
\hline \multirow[t]{4}{*}{1} & 1 & 1.1 & 0.29 & 0.15 & 6 & 0.14 & 0.04 & 6 \\
\hline & & 1.1 & $0.27^{\mathrm{b})}$ & 0.07 & 3 & 0.17 & 0.03 & 3 \\
\hline & 2 & 1.1 & 0.26 & 0.07 & 4 & - a) & - & - \\
\hline & & 1.1 & $0.32^{\mathrm{b}}$ & 0.13 & 5 & 0.11 & 0.05 & 2 \\
\hline 2 & 1 & 0.92 & 0.25 & 0.08 & 4 & 0.088 & 0.042 & 2 \\
\hline 3 & 1 & 1.1 & 0.25 & 0 & 2 & 0.21 & 0.04 & 2 \\
\hline \multirow[t]{3}{*}{4} & 1 & 0.69 & 0.11 & 0.05 & 4 & - & - & - \\
\hline & & 0.69 & $0.089^{h)}$ & 0.037 & 7 & 0.039 & 0.019 & 2 \\
\hline & & 0.69 & $0.076^{\circ}$ & 0.009 & 4 & 0.043 & 0.016 & 3 \\
\hline \multirow[t]{5}{*}{5} & 1 & 0.92 & 0.22 & 0.03 & 8 & 0.094 & 0.045 & 5 \\
\hline & & 0.92 & $0.19^{\mathrm{h}}$ & 0.06 & 7 & 0.068 & 0.034 & 5 \\
\hline & 2 & 0.92 & 0.20 & 0.06 & 6 & 0.091 & 0.041 & 2 \\
\hline & 3 & 0.92 & 0.14 & 0.04 & 3 & 0.098 & 0.023 & 3 \\
\hline & 4 & 0.92 & 0.20 & 0.02 & 2 & 0.12 & 0.01 & 2 \\
\hline \multirow[t]{2}{*}{6} & 1 & 1.1 & 0.28 & 0.13 & 7 & 0.17 & 0.04 & 6 \\
\hline & 2 & 1.1 & 0.26 & 0.07 & 11 & 0.12 & 0.05 & 6 \\
\hline 7 & 1 & 0.68 & 0.11 & 0.01 & 5 & - & - & - \\
\hline \multicolumn{9}{|c|}{ Hydrochloric acid solution } \\
\hline 1 & 1 & 0.69 & 0.046 & 0.021 & 6 & 0.028 & 0.011 & 2 \\
\hline \multirow[t]{6}{*}{2} & 1 & 1.25 & 0.42 & 0.06 & 5 & 0.076 & 0.041 & 3 \\
\hline & & 1.22 & $0.45^{b)}$ & 0.14 & 5 & 0.13 & 0.04 & 4 \\
\hline & & 1.16 & $0.25^{c)}$ & 0.06 & 5 & 0.080 & 0.018 & 4 \\
\hline & 2 & 1.25 & 0.43 & 0.12 & 5 & 0.085 & 0.067 & 4 \\
\hline & & 1.22 & $0.56^{\mathrm{h}}$ & 0.08 & 5 & 0.097 & 0.061 & 3 \\
\hline & & 1.16 & $0.53^{\circ}$ & 0.23 & 5 & - & - & - \\
\hline \multirow[t]{4}{*}{3} & 1 & 1.06 & 0.34 & 0.13 & 6 & 0.079 & 0.025 & 5 \\
\hline & & 1.06 & $0.28^{b)}$ & 0.09 & 6 & 0.078 & 0.026 & 5 \\
\hline & 2 & 1.06 & 0.21 & 0.09 & 6 & 0.032 & 0 & 2 \\
\hline & & 1.06 & $0.24^{\mathrm{b}}$ & 0.09 & 6 & 0.053 & 0.026 & 5 \\
\hline \multirow[t]{3}{*}{4} & 1 & 0.92 & 0.20 & 0.09 & 6 & 0.039 & 0.001 & 3 \\
\hline & & 0.92 & $0.19^{b)}$ & 0.06 & 6 & 0.034 & 0.009 & 3 \\
\hline & & 0.90 & $0.16^{\circ)}$ & 0.04 & 6 & 0.026 & 0.013 & 3 \\
\hline \multirow[t]{5}{*}{5} & 1 & 0.83 & 0.093 & 0.012 & 3 & 0.084 & - & 1 \\
\hline & 2 & 0.83 & 0.108 & 0.033 & 4 & 0.027 & 0.009 & 4 \\
\hline & & 0.83 & $0.066^{b)}$ & 0.004 & 3 & 0.024 & 0.007 & 3 \\
\hline & 3 & 0.69 & 0.057 & 0.006 & 2 & - & - & - \\
\hline & & 0.69 & $0.094^{b)}$ & 0.021 & 2 & - & - & - \\
\hline
\end{tabular}

a) No growth in the $c$ direction observed in any of the crystals.

b) Growth rate measured during the second time interval.

c) Growth rate measured during the third time interval. 
growth kinetics; only a slight difference in growth rates in the $\boldsymbol{a}$ direction for the two solvents but a significant increase in $c$ direction growth in phosphoric acid solution as compared to hydrochloric acid solution.

It is interesting to explore the differences between the two solvents that might explain the observed changes in crystal growth rate and morphology. The first possibility is a change in solubility with solvent. However, the solubility of phenytoin was measured in the two solvents and no significant difference was found from the reported value of $18.4 \mu \mathrm{g} / \mathrm{ml}$ in phosphate buffer.

One point to consider is the different buffering capacity of the two solvents. During crystal growth of a weak acid such as phenytoin, incorporation of growth units into the lattice could result in a depletion of hydrogen ions at the solid/solvent interface. The hydrogen ions would be removed by the reaction $\mathrm{H}^{+}+(\mathrm{DPH})^{-} \rightleftarrows(\mathrm{DPH})$ which occurs when ionized phenytoin molecules, (DPH) ${ }^{-}$, are incorporated into the crystal lattice. In the phosphoric acid solution, the change in hydrogen ion concentration would be compensated for by a shift in the reaction: $\mathrm{H}_{3} \mathrm{PO}_{4} \rightleftarrows \mathrm{H}^{+}+\mathrm{H}_{2} \mathrm{PO}_{4}^{-}$. However, in the hydrochloric acid solution, diffusion of $\mathrm{H}^{+}$from the bulk would be necessary. Thus, it is possible that the $\mathrm{pH}$ at the crystal/ solvent interface could be greater than that in the

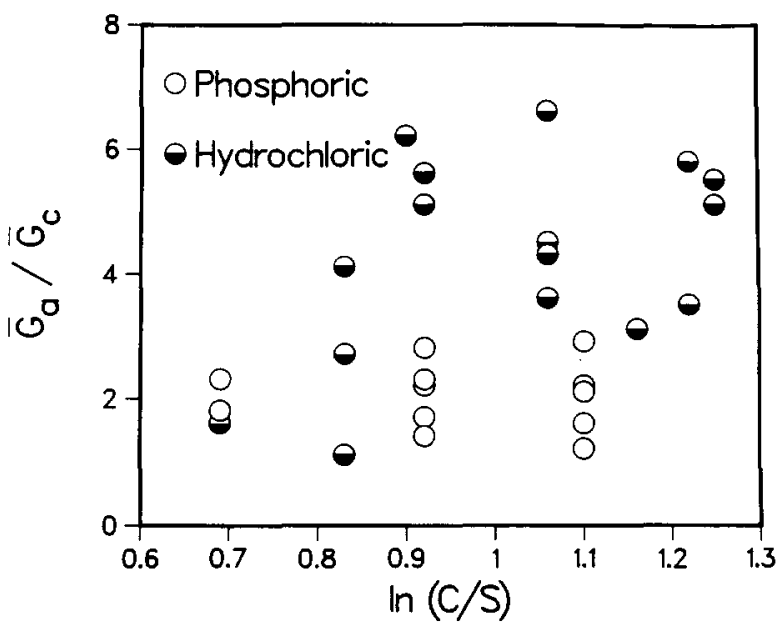

Fig. 8. The ratio of growth rates in the $a$ and $c$ direction, $\bar{G}_{a} / \bar{G}_{c}$, versus supersaturation.
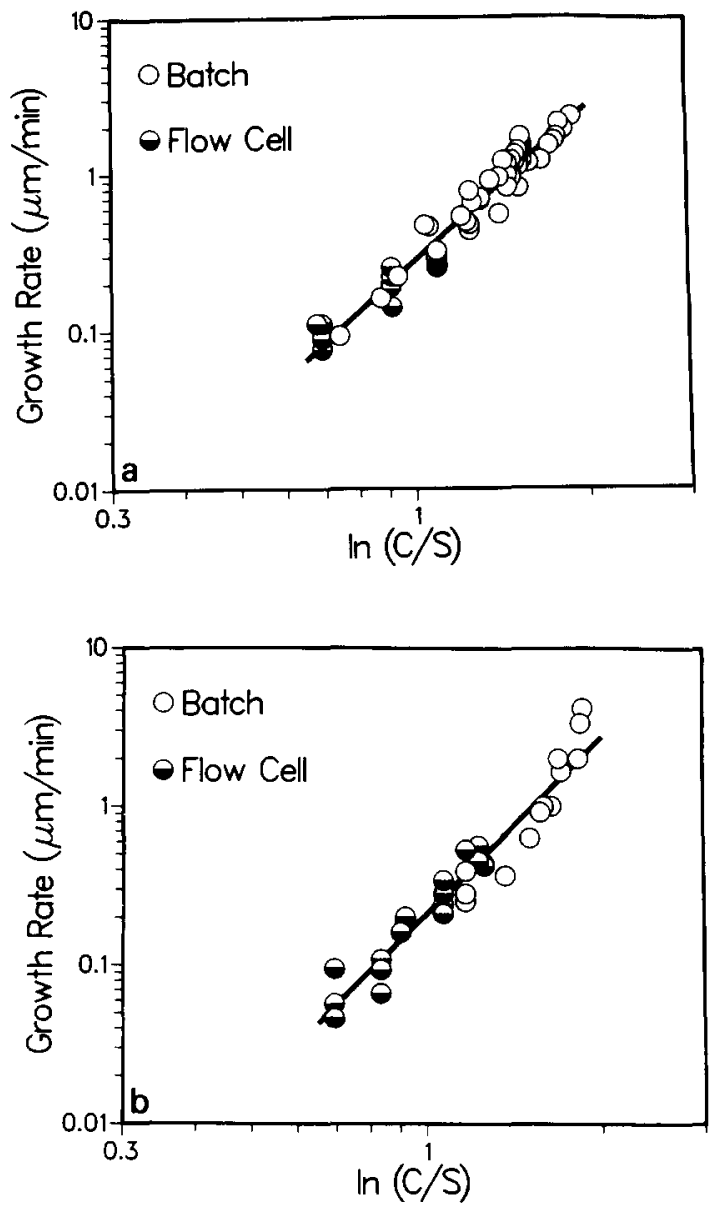

Fig. 9. Comparison of phenytoin crystal growth rates measured in batch crystallization experiments and single crystal growth in the $a$ direction: (a) phosphoric acid solution; (b) hydrochloric acid solution.

bulk solution. A higher $\mathrm{pH}$ would increase the phenytoin solubility at the interface and decrease the supersaturation at that point. This would lead to a weaker dependence of growth rate on super-

Table 2

Growth rate kinetics in pH 2.2 phosphoric acid and $\mathrm{pH} 2.2$ hydrochloric acid solutions

\begin{tabular}{lll}
\hline Solution & $\begin{array}{l}k_{\mathrm{g}} \\
(\mu \mathrm{m} / \mathrm{min})\end{array}$ & $a$ \\
\hline Phosphoric & 0.27 & 3.3 \\
Hydrochloric & 0.21 & 3.7 \\
\hline
\end{tabular}


saturation and a lower growth rate in the hydrochloric acid solution.

The existence of a localized $\mathrm{pH}$ profile at the solid/liquid interface has been used to explain the observed change in dissolution rates of weak acids and weak bases in buffered and unbuffered solutions [7-9]. However, it is not likely to be the cause of the observed differences in phenytoin crystal growth kinetics for two reasons. First, a change in $\mathrm{pH}$ can only exist when the diffusion rate of $\mathrm{H}^{+}$from the bulk to the solid/liquid interface is slow compared to the rate of removal of $\mathrm{H}^{+}$. This also implies that the hydrodynamics of the system would influence the overall kinetics by changing the diffusion boundary layer thickness. However, the rate of phenytoin crystal growth in both batch and individual crystal measurements was not influenced by such diffusive limitations. No change in kinetics was observed between batch experiments conducted at 400 and $1600 \mathrm{rpm}$ or between flow cell experiments conducted at several volumetric flow rates ( 5 to 20 $\mathrm{ml} / \mathrm{h}$ ). Second, the $\mathrm{p} K_{\mathrm{a}}$ of phenytoin is 8.06 . At pH 2.2, the fraction of ionized molecules would be extremely small and, therefore, insufficient to influence the local $\mathrm{pH}$.

Interaction between the solvent and the growing crystal faces may be altered by the different species present in the two solvents. The hydrochloric acid solution contains the species: $\mathrm{H}^{+}$, $\mathrm{Na}^{+}, \mathrm{Cl}^{-}, \mathrm{OH}^{-}$and $\mathrm{H}_{2} \mathrm{O}$. In addition to these, at $\mathrm{pH} \mathrm{2.2,} \mathrm{the} \mathrm{phosphate} \mathrm{molecules} \mathrm{are} 50 \%$ ionized and the species present are $\mathrm{H}_{3} \mathrm{PO}_{4}$ and $\mathrm{H}_{2} \mathrm{PO}_{4}^{-}$. Strong interaction between the phosphate ions and the growing crystal surfaces would be expected to hinder rather than accentuate growth rates. However, phosphate ions may influence the solution structure such that enhancement of growth rates could be possible when they are present. Finally, the existence of different impurities introduced with the two acids affecting growth rates cannot be ruled out. Trace concentrations of an impurity in the hydrochloric acid solution that strongly absorbs to crystal kink sites would be sufficient to reduce growth rates.

\section{Conclusions}

Phenytoin crystal growth rates have been equivalently measured in batch crystallization experiments with a Coulter Counter, and in a flow cell system by individual crystal observations. The results from both methods can be combined to produce a more detailed explanation of the measured growth kinetics. The batch experiments provide an average growth rate from a large number of crystals, while the individual crystal measurements permit observation of morphology, surface roughness and relative growth rates in different directions. The change in surface morphology of faces growing in the $c$ direction and the higher $c$ direction growth rate dependence on supersaturation, found when phenytoin crystals were grown in phosphoric acid solution, may indicate that phosphate ions play an important role in phenytoin crystal growth kinetics.

\section{Acknowledgments}

This work was partially supported by the National Science Foundation (Grant RII-8613745) and the Pharmaceutical Manufacturers Association Foundation (Advanced Predoctoral Fellowship in Pharmaceutics).

\section{References}

[1] B.R. Salem, R.L. Yost, G. Torosain, F.T. Davis and B.J. Wilder, Drug Intell. Clin. Pharm. 14 (1980) 605.

[2] N. Giacona, J.L. Bauman and J.K. Siepler, Am. J. Hosp. Pharm. 39 (1982) 630.

[3] R.R. Carmichael, C.D. Mahoney and L.P. Jeffrey, Am. J. Hosp. Pharm. 37 (1978) 45.

[4] P.A. Schwartz, C.T. Rhodes and J.W. Cooper, J. Pharm. Sci. 66 (1977) 994.

[5] L. Li and N. Rodríguez-Hornedo. J. Crystal Growth 121 (1992) 33.

[6] C. Misra and E.T. White, Chem. Eng. Progr. Symp. Ser. 67 (1971) 53.

[7] K.G. Mooney, M.A. Mintun, K.J. Hemmelstein and V.J. Stella, J. Pharm. Sci. 70 (1981) 13.

[8] K.G. Mooney, M.A. Mintun, K.J. Hemmelstein and V.J. Stella, J. Pharm. Sci. 70 (1981) 22.

[9] D.P. McNamara and G.L. Amidon, J. Pharm. Sci. 75 (1986) 858. 